\title{
Activités humaines reflétées dans les sols d'unités d'habitation contemporaine et préhispanique du Yucatan (Mexique) : études chimiques, ethnoarchéologiques et archéologiques \\ Luis Barba, Fabienne de Pierrebourg, Claudia Trejo, Agustín Ortiz, Karl Link
}

\section{Résumé}

L'archéologie de l'habitation et la recherche d'aires d'activité connaissent une ampleur croissante et l'analyse chimique des sols s'est révélée extrêmement utile pour déterminer les espaces où se réalisaient des activités humaines spécifiques. Le travail présenté ci-dessous envisage une analyse comparative de deux unités d'habitation, l'une abandonnée trente ans avant les fouilles et l'autre préhispanique. Basé également sur une étude ethnoarchéologique, il montre que la juxtaposition de données archéologiques et de dosages géochimiques variés permet de déterminer l'articulation fonctionnelle de l'espace domestique et d'identifier des aires d'activité.

\section{Abstract}

The interest of household archaeology and activity areas research has grown in the last few years. The use of chemical analysis of floors as a tool has provided significant information for detecting the space where specific human activities occur. This paper is a comparative study of two Maya households ; the first one, abandoned thirty years ago and the other, of Prehispanic date. The correlation of various chemical and archaeological data helps in determinating the functional articulation of domestic space and activity areas.

\section{Citer ce document / Cite this document :}

Barba Luis, Pierrebourg Fabienne de, Trejo Claudia, Ortiz Agustín, Link Karl. Activités humaines reflétées dans les sols d'unités d'habitation contemporaine et préhispanique du Yucatan (Mexique) : études chimiques, ethnoarchéologiques et archéologiques. In: Revue d'Archéométrie, n¹9, 1995. pp. 79-95;

doi : https://doi.org/10.3406/arsci.1995.929

https://www.persee.fr/doc/arsci_0399-1237_1995_num_19_1_929

Fichier pdf généré le 07/01/2019 


\title{
ACTIVITES HUMAINES REFLETEES DANS LES SOLS D'UNITES D'HABITATION CONTEMPORAINE ET PREHISPANIQUE DU YUCATAN (Mexique): Etudes chimiques, ethnoarchéologiques et archéologiques
}

\author{
Luis BARBA*, Fabienne de PIERREBOURG**, Claudia TREJO*, Agustín ORTIZ* et Karl LINK*
}

\begin{abstract}
Résumé: L'archéologie de l'habitation et la recherche d'aires d'activité connaissent une ampleur croissante et l'analyse chimique des sols s'est révélée extrêmement utile pour déterminer les espaces où se réalisaient des activités humaines spécifiques. Le travail présenté ci-dessous envisage une analyse comparative de deux unités d'habitation, l'une abandonnée trente ans avant les fouilles et l'autre préhispanique. Basé également sur une étude ethnoarchélogique, il montre que la juxtaposition de données archéologiques et de dosages géochimiques variés permet de déterminer l'articulation fonctionnelle de l'espace domestique et d'identifier des aires d'activité.
\end{abstract}

\begin{abstract}
The interest of household archaeology and activity areas research has grown in the last few years. The use of chemical analysis of floors as a tool has provided significant information for detecting the space where specific human activities occur. This paper is a comparative study of two Maya households; the first one, abandoned thirty years ago and the other, of Prehispanic date. The correlation of various chemical and archaeological data helps in determinating the functional articulation of domestic space and activity areas.
\end{abstract}

Mots-clés: Analyse chimique des sols, aires d'activité, ethnoarchéologie, archéologie, Yucatán, Mexique.

Key-words: Chimical analysis, floors, activity areas, ethnoarchaeology, archaeology, Yucatan, Mexico.

L'archéologie de l'habitation connaît un intérêt croissant au Mexique et dans l'aire maya. Il s'agit de retrouver, dans les vestiges de l'espace domestique, les activités qui s'y déroulaient et les acteurs qui y prenaient part. Pour cela, l'archéologue dispose des vestiges de la culture matérielle - morphologie générale de l'espace, caractéristiques architecturales, localisation des bâtiments et distribution des artefacts. A partir de ces éléments, il essaie de déterminer des aires et des espaces d'activités. Cependant, certaines activités domestiques ne laissent pas de traces matérielles, d'autres déposent des vestiges périssables ou difficilement identifiables. Le recours à une analyse chimique des sols est susceptible de combler l'absence de témoins matériels par la mise en évidence des composants chimiques déposés dans le sol par la répétition d'activités en un même lieu. En effet, il apparaît, à travers l'étude de deux unités d'habitation de l'aire maya septentrionale, l'une abandonnée récemment et l'autre préhispanique, que la corrélation des données archéologiques et chimiques permettent d'identifier les limites de l'espace domestique, son articulation fonctionnelle et mettent en evi- dence des aires d'activité.

Les travaux présentés ci-dessous sont les résultats de deux recherches sur les possibilités d'interprétations des vestiges domestiques matériels et chimiques. Une étude ethnoarchéologique sur l'habitation maya a montré que la morphologie de l'espace domestique, des bâtiments et la distribution de la culture matérielle étaient porteurs d'informations sur l'articulation fonctionnelle de l'espace, sur la vie domestique et sur les modes d'occupations (Pierrebourg, 1989, 1994/95). Les autres recherches, menées par le Laboratoire de Prospection Archéologique de l'Institut de Recherches Anthropologiques (Université Nationale Autonome de Mexico) et dirigées par Luis Barba, ont démontré que la distribution des composants chimiques déposés dans les sols d'habitations n'est pas aleatoire mais en relation avec les activités humaines se déroulant dans l'enceinte domestique (Barba et Manzanilla 1987; Manzanilla et Barba, 1990; Barba et Ortiz, 1992). L'étude d'une habitation de Muxucuxcab (Yucatán) abandonnée trente ans avant les fouilles est purement méthodologique. Elle représente une étape intermédiaire entre les tra-

\footnotetext{
* Instituto de Investigaciones Antropológicas, Universidad Nacional Autónoma de México, Ciudad Universitaria, Circuito Exterior, Delegación Coyoacán MEXICO, D. F. CP. 04510. Mexique.

** Université de Paris I-Panthéon-Sorbonne, Centre de Recherches en Archéologie Précolombienne, 3 rue Michelet, 75006 PARIS.
} 


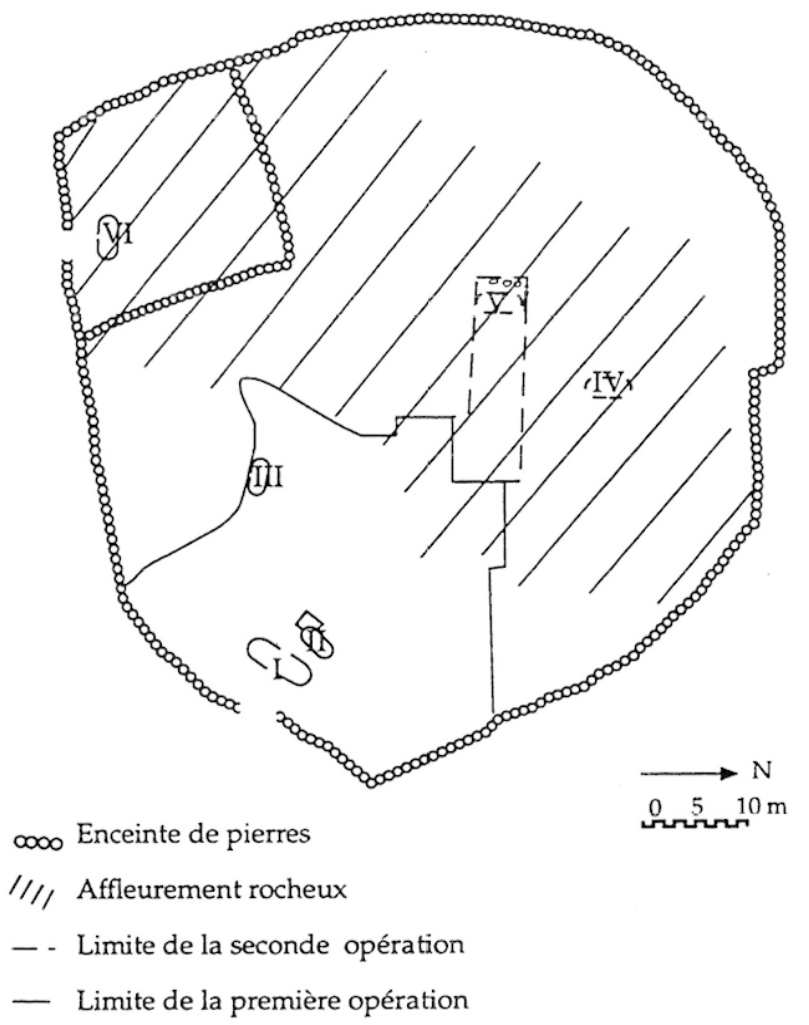

Fig. 1: Muxucuxcab, configuration générale du lot domestique et limites de l'espace étudié.

vaux réalisés dans des habitations occupées (Barba et Ortiz, 1992) et leurs applications archéologiques. Il s'agit de contrôler la formation des vestiges et de tester une méthode d'analyse chimique des sols dans un contexte archéologique mais connu. En effet, les vestiges considérés proviennent d'une habitation tout à fait comparable à celles préalablement étudiées dans un village voisin (Pierrebourg, 1994/95). De plus, certaines interprétations ont été confirmées par le témoignage d'un habitant de Muxucuxcab. La confrontation des données archéologiques et des données chimiques ayant donné des résultats tout à fait satisfaisants, la même méthode a été appliquée aux vestiges de la Plate-forme du Cabrío, habitation préhispanique de Kabah (Yucatán).

\section{METHODE D'ANALYSE DES SOLS}

Depuis une soixantaine d'années, en Europe (Arrhenius, 1931) comme aux Etats Unis (Cook et Heizer, 1965), les archéologues essaient de palier à la disparition des témoins matériels par l'analyse chimique des sols. Nous ne referons pas icil'historique des techniques employées; pour cela, il est possible de se référer au résumé de leurs succès et de leurs échecs dressé par Keeley (1981) et à l'article proposé par Mejia et Barba (1988). Le dosage des teneurs en phosphates des sols a été particulièrement utilisé. Il a permis de localiser des sites archéologiques (Hamond, 1983), de déterminer différentes zones d'activités existantes sur un site, d'identifier des aires de cultures, de localiser l'emplacement de sépultures (Craddock, 1982) et de définir des straté- gies de fouilles Provan (1971).

Selon Luis Barba et son équipe, les analyses chimiques des sols sont particulièrement appropriées à la recherche d'informations sur les activités ayant été réalisées sur ces sols à une échelle plus petite et notamment dans des unités d'habitation. Il s'agit, à travers l'étude de composantes chimiques variés de mettre en évidence des aires ou des espaces d'activités. Cette méthode a été mise au point à la suite de divers travaux réalisés dans des habitations occupées, le plus notable étant celui réalisé dans deux habitations de Xiloxochitla, Tlaxcala (Barba et Ortiz, 1992). Des relations existantes entre la distribution des composants chimiques et les activités domestiques ont ainsi pu être mises en évidence. Puis elle a été appliquée à diverses contextes archéologiques (Barba, 1986; Barba et Manzanilla, 1987; Manzanilla et Barba, 1990; Ortizet Barba, 1993; Ortizet al., 1994). Les activités interprétables sont celles qui étaient pratiquées quotidiennement et, durant une longue période, à un même endroit ou celles, plus sporadiques, qui mettaient en oeuvre un matériel assez abondant pour contaminer le sol de manière significative. Ces activités déposent dans les sols des composants chimiques susceptibles d'être décelables après plusieurs siècles et elles produisent des changements intenses des contenus chimiques des sols. Aussi, seules les grandes différences de concentrations qui peuvent être mises en évidence par des techniques simples apportent des informations archéologiques. Ceci a permis à Luis Barba et à son équipe de développer une méthode simple, peu coûteuse et rapide qui facilite le traitement d'un grand nombre d'échantillons tout en étant accessible à de nombreux projets.

Les composants chimiques considérés dans les études présentées ci-dessous sont: les phosphates, les carbonates et le potentiel hydrogène ainsi que les variations de couleurs, la présence d'albumine et d'acides gras dans le cas des sols de la Plate-forme du Cabrío. Les couleurs sont déterminées en référence à la table Munsell, système universel et normalisé, dont les coordonnées ont été modifiées afin de faciliter le traitement informatique (Barba, 1989). Leurs variations sont susceptibles de refléter des aires de cuisson, la présence de matière organique, la diversité des matériaux de construction et d'éventuelles réparations. L'augmentation du taux de carbonates est liée à l'utilisation de pierres calcaires et à la présence de chaux. La chaux est principalement utilisée dans la construction mais elle est également ajoutée à l'eau de cuisson du maïs. La détermination des carbonates se fait par réaction effervescente à l'acide chlorhydrique dont les différents niveaux sont répartis sur une échelle de 0 à 5 (1) (Barba et Ortiz, 1992). Selon la procédure habituelle, le potentiel hydrogène est mesuré à l'aide d'un pH mètre muni d'une électrode courte. Ses valeurs maximales indiquent la présence d'hydroxydes provenant de cendres. L'augmentation des taux de phosphates signale la présence de matières organiques; en particulier, elle indique la localisation des aires de décharge et d'élevage. Les méthodes de dosage de phosphates sont discutées. Certains (Craddock, 1982; Eidt, 1973) sont favorables aux méthodes colorimétriques; simples et peu onéreuses, elles offrent des résultats rapidement exploitables. D'autres jugent ces techniques peu satisfaisantes et préfèrent des méthodes plus

(1) Dans les figures $3,5,8,9$ et 12, les dosages de carbonates et de phosphates, résultats de test semi-quantitatifs, sont caractérisés par des échelles comparatives. Dans d'autres travaux, le laboratoire a mis au point une courbe de calibrage permettant de convertir ces données à des quantités absolues de concentration. Mais, dans le cas des analyses présentées ici, il n'était pas nécessaire d'obtenir des quantités exactes, la variation des valeurs apportant les informations significatives sur les activités humaines. 


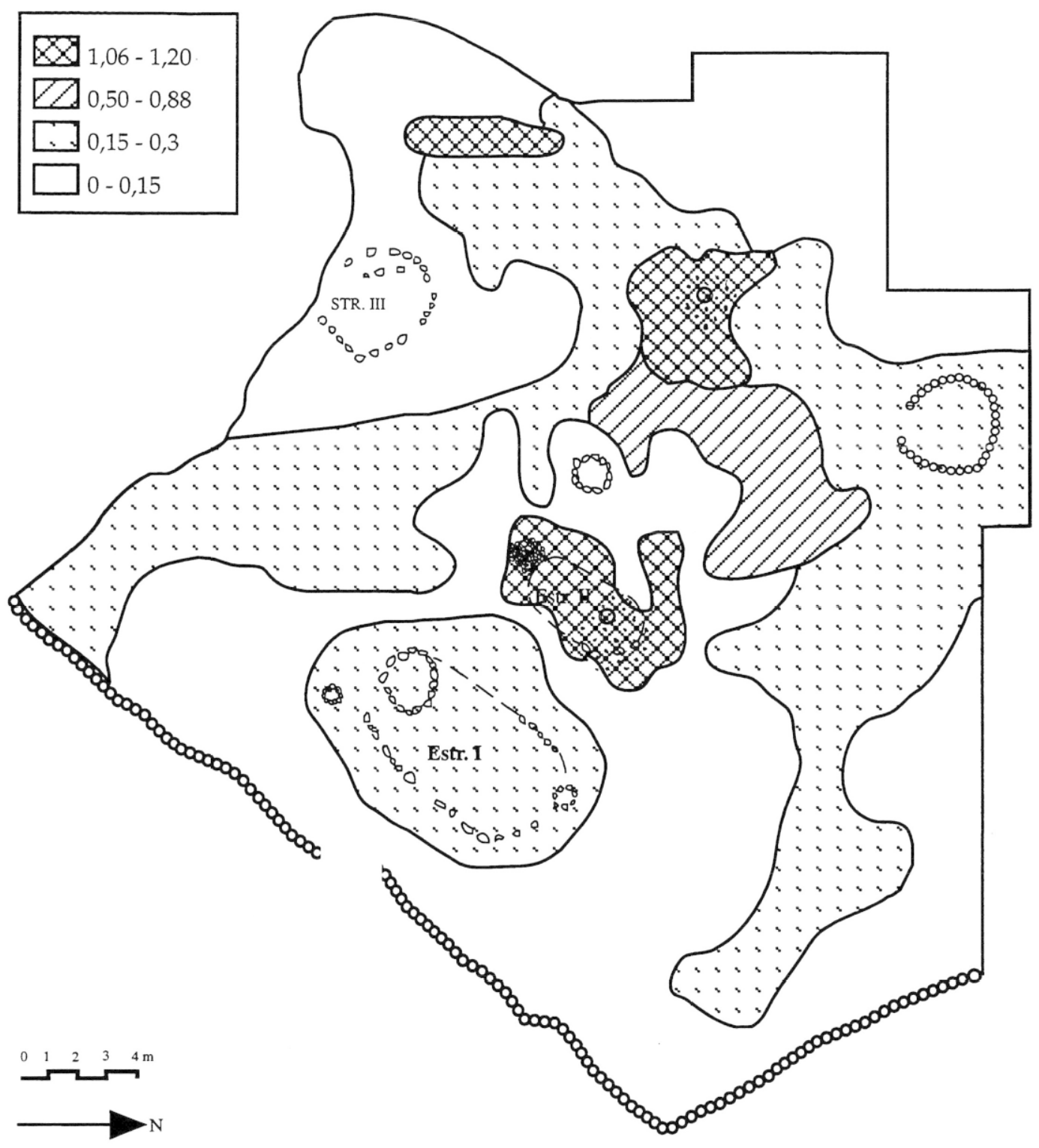

Cendres; ${ }_{8}$ Enceinte de pierres; o Citerne

Fig. 2: Muxucuxcab, densité du matériel (par $\mathrm{m}^{2}$ ).

sophistiquées, plus sûres mais plus lourdes et peu accessibles aux laboratoires de sciences humaines. Par exemple, le phosphore peut être dosé par spectrophotocolorimétrie ou par des méthodes potentiométriques par électrode spécifique (Guillerault et Bazile, 1987). Pour les raisons exposées antérieurement (recherche de grandes variations de concentrations par une méthode rapide et peu onéreuse), Luis Barba et son équipe ont choisi d'utiliser un test semi-quantitatif. Il est réalisé à l'aide d'un colorimètre: la quantité de phosphates contenue dans un échantillon est mise en évidence par l'intensité de la couleur bleue apparaissant à la surface d'un papier filtre (2). La présence d'albumine est établie par la libération d'ammoniac qui est détectée par un changement de couleur sur un papier indicateur. L'albumine est présente dans les tissus, les oeufs, le lait et le sang; elle indique des aires de préparation alimentaire et de consommation. La présence d'acides gras est estimée par la formation de savons d'ammonium: la quantité et la persistance des bulles produites étant proportionnelles à la quantité d'acides gras contenue dans l'échantillon. Ceux-ci apparaissent dans les fermentations, les savons, les graisses animales et végétales; ils signalent également des aires de préparation et de consommation (Barba et al., 1991).

\section{EXEMPLE ETHNOARCHEOLOGIQUE}

Muxucuxcab est situé dans le tiers nord de la péninsule du Yucatán; plate-forme massive, partiellement immergé, elle est composée de roches calcaires et salines (gypse) pliocènes et miocènes. Sa structure tabulaire, la géologie calcaire et des pluies abondantes ont développé une topographie de nature karstique ayant engendré un réseau hydrographique souterrain. Les sols de cette région se composent principalement de nitosoles eutriques et lithosols (3). Le climat est tropical $\sec (\mathrm{A3}$ a, selon la classification FAO-UNESCO), le

(2) Voir note 1.

(3) Selon la classification de FAO-UNESCO (1975) et la correspondance avec la terminologie maya établie par Dunning (1991). 

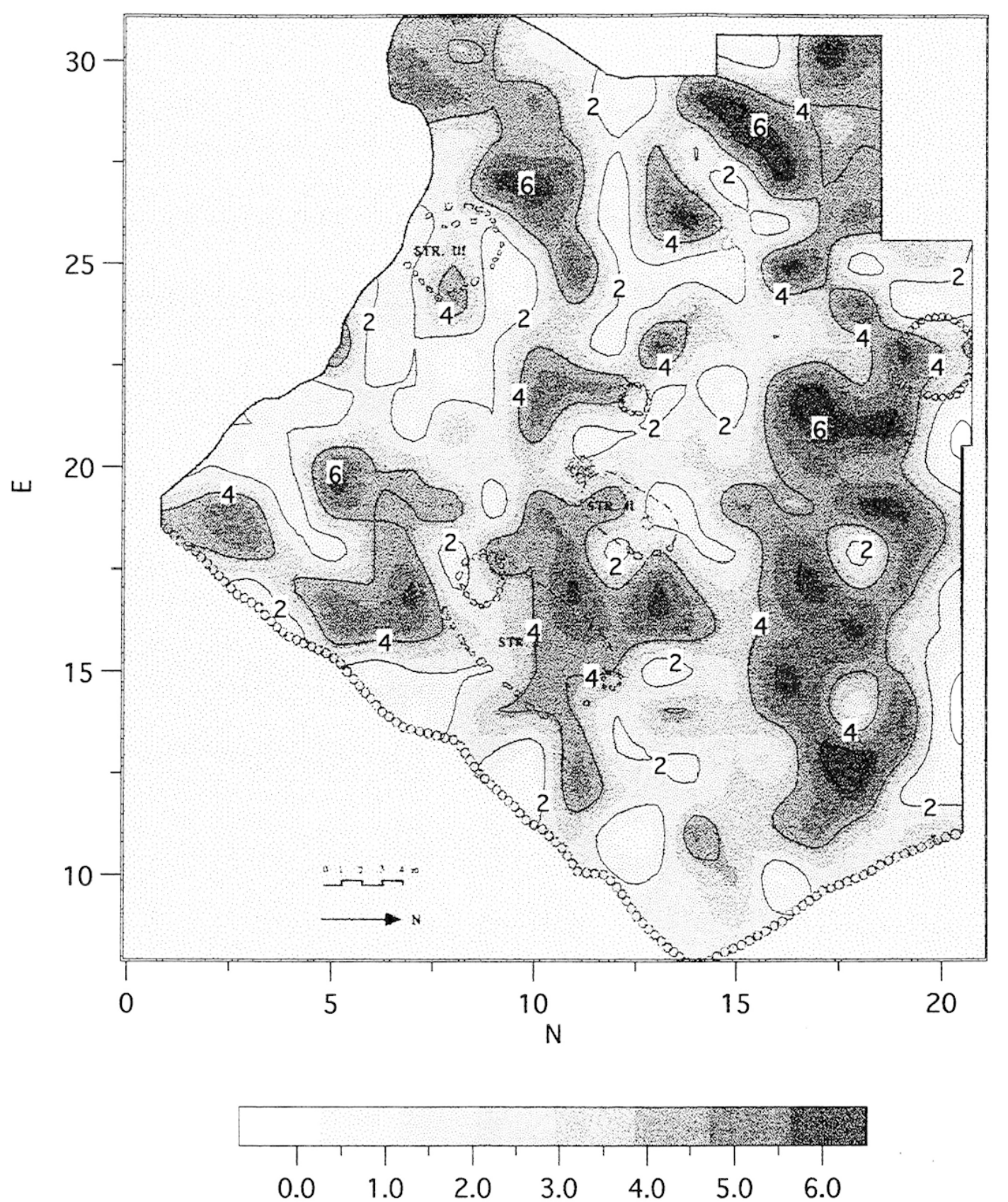

Fig. 3: Muxucuxcab, distribution des valeurs de phosphates.

total des pluies annuelles est compris entre 500 et $1000 \mathrm{~mm}$. Comme toutes les habitations de cette région, celles de Muxucuxcab se composent d'un ou de plusieurs bâtiments de forme elliptique ne présentant pas de division interne. Chaque bâtiment remplit une fonction déterminée (logis, cuisine, réserve). Leurs parois sont faites de rondins et leur toit de palmes ou de chaume. Après leur destruction, il ne reste que le sol constitué d'un remblai de pierres et de terre, haut de 10 à $50 \mathrm{~cm}$, retenu par une rangée de pierres. Les bâtiments sont situés dans un espace clos par un muret de pierres sèches où se déroulent diverses activités domestiques, où les plantes médicinales, comestibles ou ornementales sont cultivées et où les animaux domestiques sont élevés. L'espace clos de l'unité domestique qui nous intéresse (fig. 1) est particulièrement grand $\left(4723 \mathrm{~m}^{2}\right)$. Il est traversé par une élévation rocheuse, haute de $3,5 \mathrm{~m}$, qui représente la limite de l'espace où se déroulaient les activités réalisées par les habitants des structures I et II.
L'étude présentée ici est centrée sur cet espace; les structures voisines ont été considérées uniquement dans le but de le délimiter. Les vestiges d'occupation étant superficiels, leur décapage a été limité à un nettoyage de surface. Le matériel a été collecté sur toute l'aire considérée en fonction d'un quadrillage dont l'unité de base était de $4 \mathrm{~m}^{2}$. Les échantillons de sol ont été prélevés tous les deux mètres à l'extérieur et tous les mètres dans les structures.

En termes généraux, les vestiges matériels (fig. 2) et les valeurs chimiques (fig. 3 à 5) présentent une même distribution qui reflètent les limites de l'espace domestique utilisé par les habitants des structures I et II ainsi que son articulation fonctionnelle. Les limites de cet espace sont marquées, à l'est, par un mur de pierres sèches et, dans les autres directions, par la diminution de la densité du matériel et des valeurs chimiques. En effet, l'absence de contamination matérielle et chimique correspond à l'espace laissé en friche et dénué 
d'activités qui est présent dans toutes les habitations de cette région. Au nord-ouest, au pied de l'affleurement rocheux, une forte contamination du sol est associée à un matériel ancien (1500- 1800 ap. J. -C.); elle correspond à l'occupation de la str. $\mathrm{V}$ située au sommet de l'élévation (fig. 1). L'espace domestique quotidiennement utilisé est identifiable par l'augmentation des composants chimiques imprégnés dans le sol et leur variation de densité reflète la succession des trois espaces caractéristiques de l'habitation yucatèque (Pierrebourg, 1989, 1994/95). Au centre, une aire de fortes valeurs chimiques associées à un matériel abondant correspond aux espaces couverts et à leurs abords immédiats. Cette aire est entourée par un espace relativement propre et peu contaminé. C'est l'espace résidentiel, régulièrement balayé, où se déroulent des activités peu salissantes: se tenir, circuler, égrener le maiis, coudre, par exemple. Il est bordé par l'espace périphérique présentant une forte densité de matériel et des taux de phosphates élevés où se déroulent la plupart des activi- tés contaminantes. On y trouve, entre autre, les aires de lavage, de décharge et d'élevage. Plus en détail, la distribution du matériel et celle des valeurs chimiques permettent d'identifier diverses aires d'activité à l'intérieur des espaces couvert, résidentiel et périphérique.

L'espace couvert se compose d'un logis et d'une cuisine. Les activités réalisées dans les logis laissent peu de traces. Les principales sont: dormir dans un hamac, conserver et ranger dans des sacs, des paniers, des caisses ou par suspension et éventuellement cuisiner. Le logis (str. I) est reconnaissable par sa localisation, face à la rue, et par ses grandes dimensions mais très peu de vestiges matériels y étaient associés. Aussi, quelques aires d'activité n'ont pu être déduites que par l'analyse des composants chimiques imprégnés dans le sol. Des augmentations de $\mathrm{pH}$ et de phosphates apparaissent dans chaque abside (fig. 3 et 4), elles sont probablement relatives à la présence de foyers dont les trois pierres auraient été déplacées lors de l'occupation postérieure (str. III) et utilisées dans la construction des enceintes
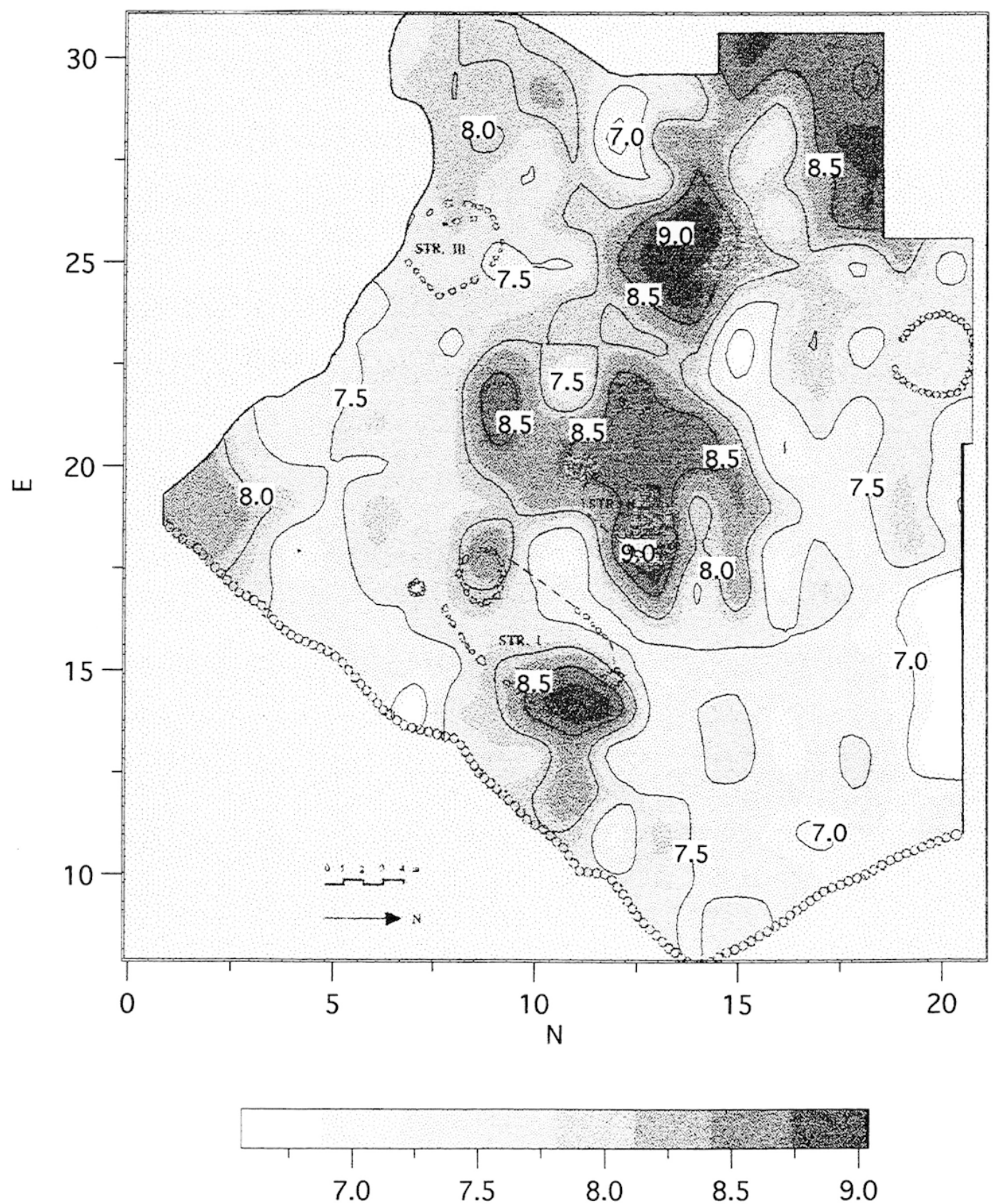

Fig. 4: Muxucuxcab, distribution des valeurs de $\mathrm{pH}$. 

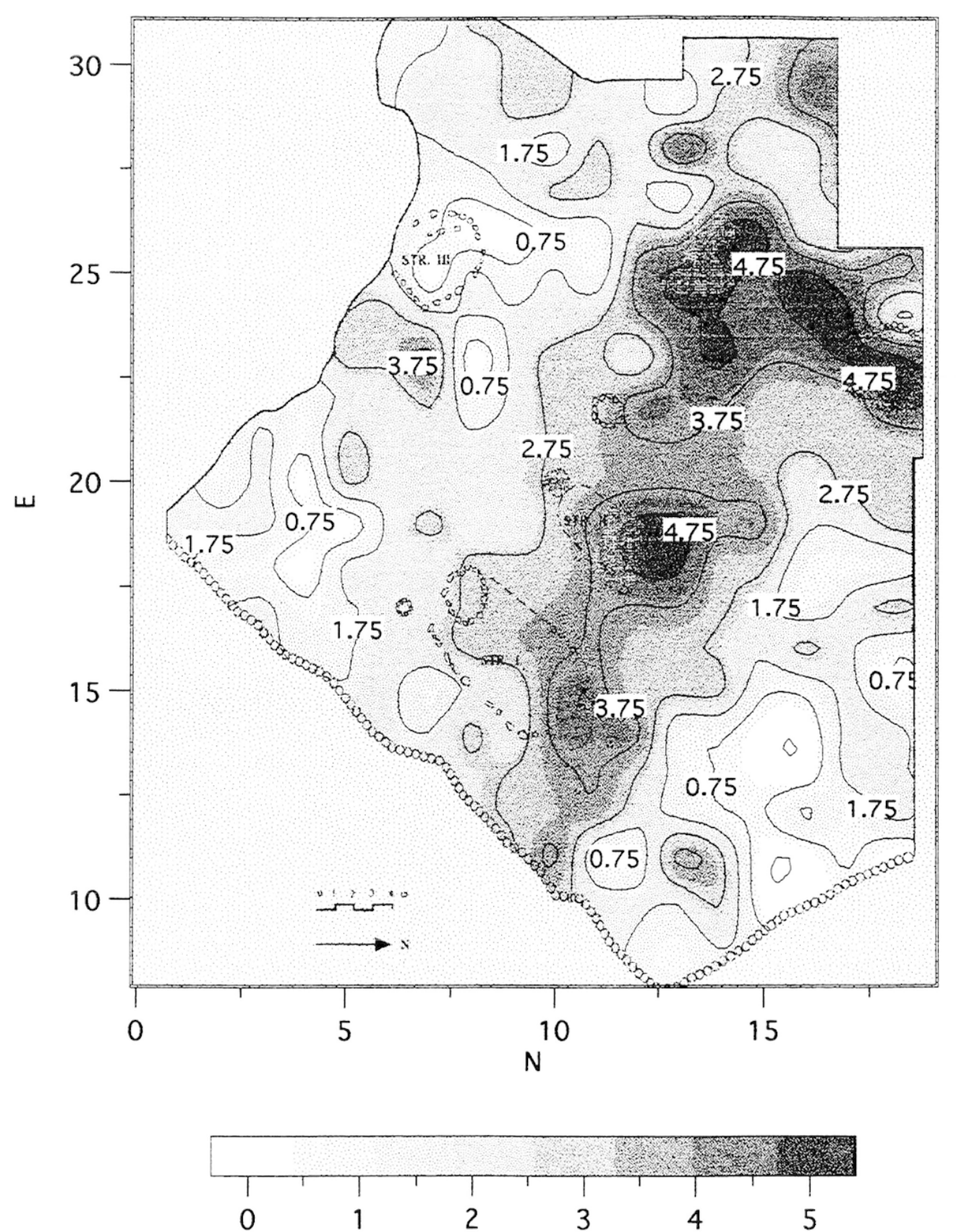

Fig. 5: Muxucuxcab, distribution des valeurs de carbonates.

protégeant les plantes fragiles. Il est, en effet, courant que des familles disposant d'une cuisine préparent certains repas dans le logis, en particulier, les petits déjeuners. Dans le cas de la str. I, le foyer nord est probablement le plus ancien. Dans cette section, une forte concentration de carbonates (fig. 5) suggère un sol refait et bien conservé sur lequel a pu être installé un grenier. En effet, Smyth (1990) a noté que les aires de conservation du mais présentent de forts taux de carbonates dus à l'ajout de chaux afin de protéger le grain des insectes. Selon notre informateur, durant les saisons froides, la famille disposait des braises sous les hamacs. Cette habitude peut expliquer les augmentations de $\mathrm{pH}$ notables au centre et au sud de la structure.

La cuisine se distingue par des taux de $\mathrm{pH}$ élevés qui reflètent l'extension des cendres du foyer sur toute sa superficie. Dans le détail, les variations internes des valeurs chimiques met en relief la division bipartite caractéristique des cuisines yucatèques et, en association avec les données archéologiques, elle permet de définir des aires d'activité dont les vestiges se prolongent vers l'extérieur. Dans la section nord, où le sol est totalement imprégné de cendres, se déroulaient les tâches de préparation culinaire et les repas y étaient consommés. Les valeurs du $\mathrm{pH}$ extrêmement fortes $(9,0)$ indiquent l'emplacement du foyer (fig. 4). Les vestiges d'une petite citerne dans laquelle était déposée l'eau de travail, situés dans la même section, ont augmenté la teneur du sol en carbonates. Cette citerne contenait de la cendre qui était ajoutée à l'eau afin de l'adoucir. De cette manière, le savon mousse normalement et conserve ses propriétés nettoyantes perdues en eau dure - contenant de fortes concentrations d'ions de calcium et de magnésium - caractéristique de la péninsule (Barba et Cordova, 1985). Comme le montre l'extension des valeurs du $\mathrm{pH}$ vers l'ouest, cette eau était probablement utilisée dans des tâches domestiques réalisées le long de la paroi postérieure et rejetée à l'extérieur. Il est, en effet, coutume de laver la vaisselle à cet endroit. L'eau de consommation était déposée 
dans des bassins posés sur une table de rondins aménagée le long de la paroi postérieure. L'autre section présente un matériel très peu abondant et une baisse des valeurs chimiques notable confirmant la rareté des activités réalisées dans la section opposée au foyer.

Notons également que la contamination des espaces couverts se prolongeant à l'extérieur reflète l'extension de certaines tâches. Par exemple, les forts taux de $\mathrm{pH}$ présents derrière la cuisine proviennent probablement du rejet des eaux de travail chargees en cendres, des cendres déposées dans la citerne ou de celles du foyer. L'extension des vestiges d'activités peut être également due à leur écoulement à travers les parois, le long de pentes ou à leur transport lié à une circulation fréquente. Par exemple, certains taux de pH élevés indiquent que les cendres des foyers s'ecoulaient à travers les parois. Les forts taux de carbonates à l'extérieur de la cuisine est le résultat - post-abandon - de la destruction de la citerne. Par ailleurs, l'extension des composants chimiques, selon un axe nord-sud, précise des axes de circulation et suggère que les deux bâtiments dispo- saient de deux portes situées au centre de leurs parois longitudinales.

La salle de bains consiste en un petit appendice situé au sud de la cuisine qui a été identifié par un remblai de pierres compact. D'un point de vue chimique, l'augmentation du $\mathrm{pH}$ indique que l'eau utilisée était également chargée de cendre et provenait de la citerne.

Finalement, il est intéressant de noter que la nature précaire de la str. III, signalée par notre informateur, est confirmée autant par la faible densité de matériel que par celle des valeurs chimiques (fig. 3 à 5).

Dans l'espace résidentiel, régulièrement balayé se déroulent des activités peu contaminantes correspondant à de faibles taux de phosphates et à une densité de matériel minime (fig. 3 et 4). Cependant, la prolongation des valeurs chimiques fortes le long d'un axe nordsud reflete l'axe de circulation habituel dans l'habitation maya allant de l'entrée vers le fond de l'espace domestique où se déroulent les activités les plus contaminantes.

Les forts taux de phosphates et l'augmentation de la

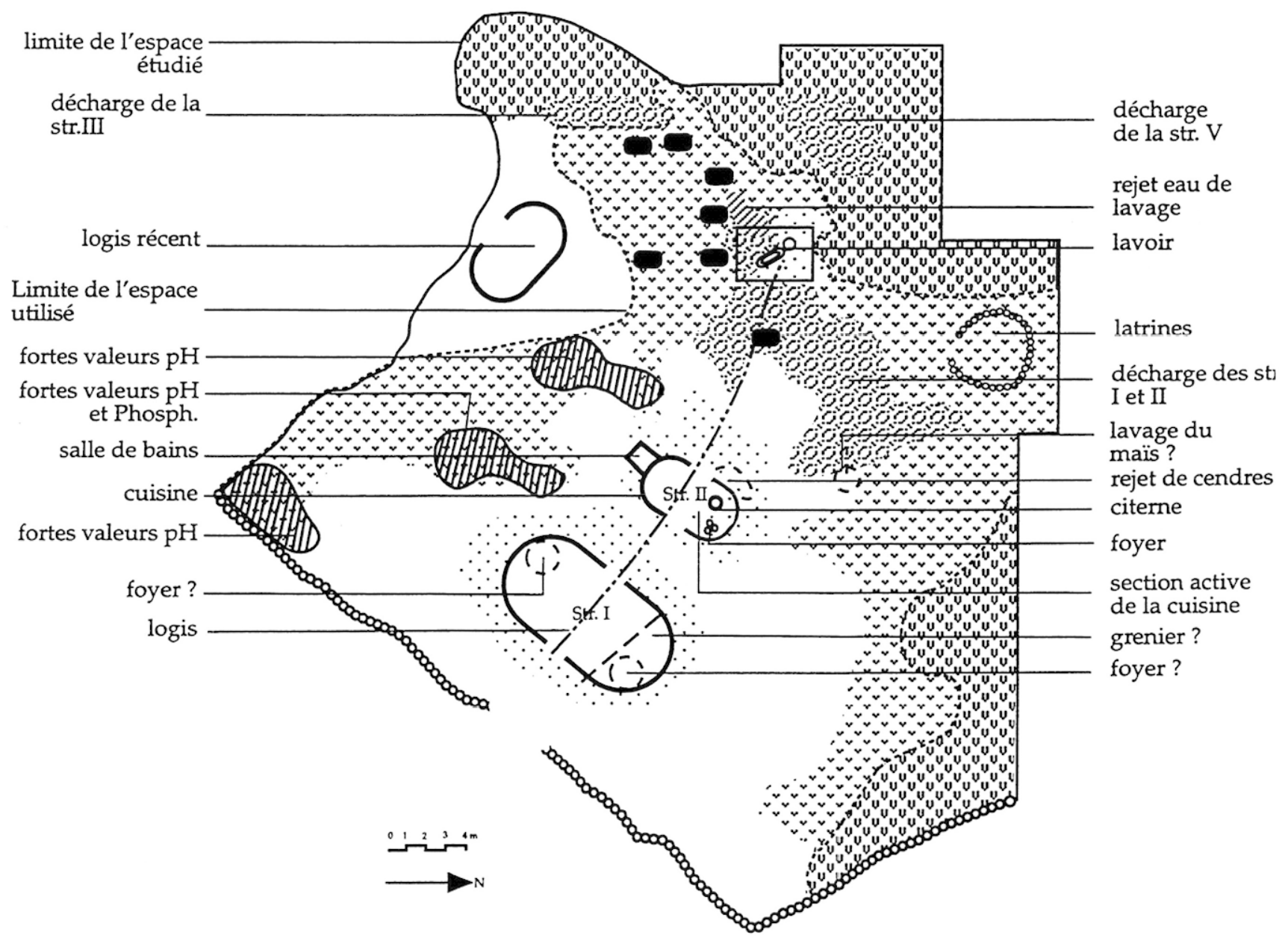

Espace résidentiel 


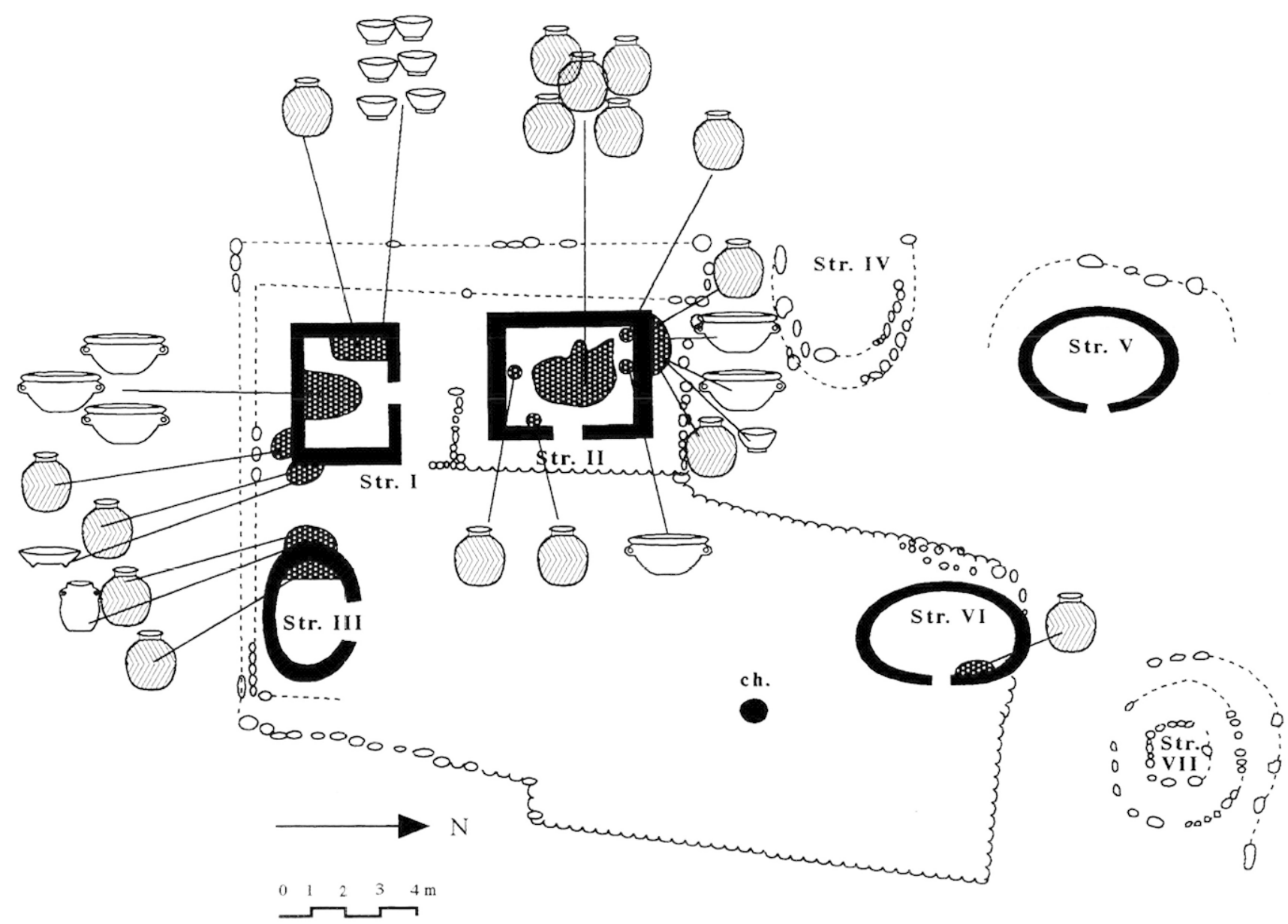

Assises de parois

Reconstruction hypothétique

ch. : citerne (chultún)

Jarre de type Yokat Strié
Bol

Jarre à puiser
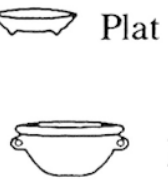

Bassin

Fig. 7: La plate-forme du Cabrío, Kabah; localisation des poteries identifiees.

densité du matériel (fig. 2 et 3 ) traduisent la concentration des activités contaminantes réalisées dans l'espace périphérique et en particulier la présence de décharges. Bien que des rejets soient observables dans tout cet espace, les plus fortes concentrations de phosphates et de matériel reflètent les aires de rejets habituelles. Une d'elle a été localisée derrière la cuisine, emplacement cohérent avec les données ethnographiques. L'augmentation de $\mathrm{pH}$ associée est due à la coutume de brûler les déchets. Une petite décharge associée à la str. III est signalé par une augmentation de phosphates et une concentration de matériel situées à l'écart. D'autres taux de $\mathrm{pH}$ élevés et dissociés des décharges indiquent la présence de fours de terre confirmée par la fouille de quelques-uns. Le lavoir a été identifié par la présence d'une petite citerne et par une grande concentration de cendres ajoutées à l'eau de lavage déposée dans la citerne et rejetée autour. La cuve était posée sur quatre poteaux fichés au sol dont nous avons retrouvé les trous. D'un point de vue chimique, cette aire d'activité se caractérise par un fort taux de pH et, à l'est des trous de poteaux, par une augmentation des phosphates probablement relative au rejet des eaux sales. Les forts taux de carbonates sont dus à la destruction de la citerne. Au nord, un enclos de pierres sèches servait de latrines, il est entouré par une augmentation de carbonates reflétant l'utilisation de chaux pour réintégrer les déchets dans le sol. Enfin, dans cet espace, comme dans l'espace précédent, de fortes valeurs de phosphates signalent la réalisation de diverses activités contaminantes ne lais- 
sant pas de traces matérielles telles que l'élevage ou des cultures intensives. Au nord-est, là où, selon notre informateur un cheval était attaché, les taux de phosphates augmentent.

Après l'étude d'habitations occupées (Barba et Ortiz, 1992), ce travail confirme la conservation des vestiges chimiques d'activités et leur pertinence pour comprendre l'articulation fonctionnelle de l'espace domestique et pour identifier des aires d'activité (fig. 6). La superposition des données chimiques et archéologiques indique clairement les limites de l'espace domestique utilisé et sa division. L'analyse chimique des sols a été particulièrement utile pour localiser l'emplacement de certaines activités n'ayant pas déposé de vestiges matériels dans le logis. Aussi, avant d'appliquer cette méthode à une habitation préhispanique, nous retiendrons les principales conclusions sur la signification des composants chimiques imprégnés dans les sols d'occupation. La présence de foyers, le maniement et le rejet de l'eau de travail chargée de cendres se traduisent par des taux de $\mathrm{pH}$ élevés. Les latrines conjuguent des hausses de car- bonates et de phosphates et les décharges un pH et des taux de phosphates élevés. Les aires de préparations alimentaires et de consommation présentent une augmentation de phosphates et les fours de terre sont identifiables par celle du pH. Enfin, les aires de circulations fréquentes apparaissent à travers l'extension des vestiges chimiques.

\section{LA PLATE-FORME DU CABRIO: UNE HABITATION PREHISPANIQUE}

La plate-forme du Cabrío est une habitation d'un site de la région Puuc, Kabah. Dans cette région au relief karstique, où alternent des collines calcaires et des dépressions fertiles d'extension variable, le réseau hydrographique est totalement souterrain et peut être situé à plus de $80 \mathrm{~m}$ de profondeur. Aussi, comme toutes les unités d'établissement, la Plate-forme du Cabrío a été construite sur un affleurement rocheux dans lequel a été creusée une citerne de $4 \mathrm{~m}$ de profondeur. Une fois la citerne creusée, l'élévation initiale aurait été égalisée
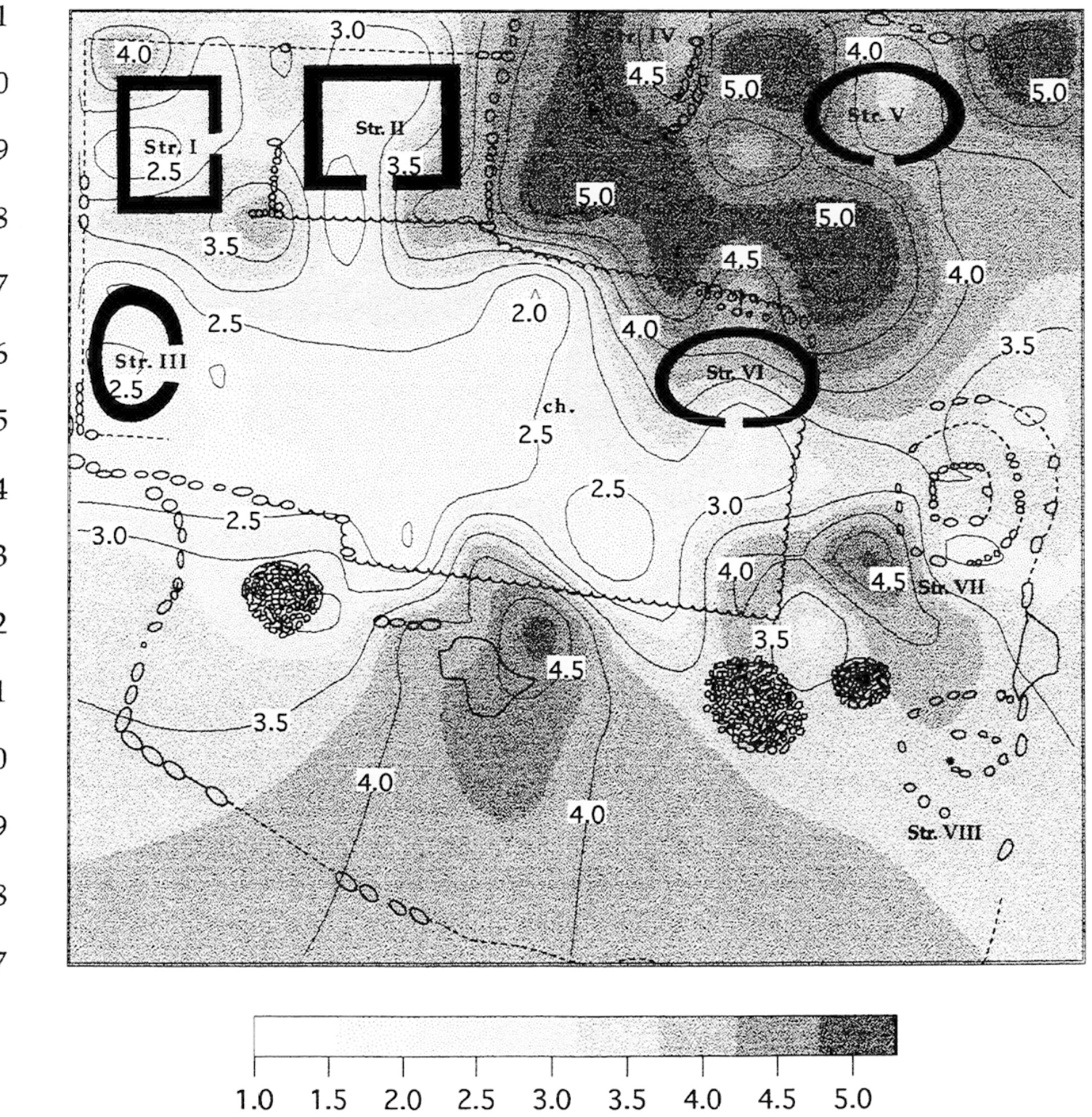

Fig. 8: La plate-forme du Cabrío, Kabah; distribution des valeurs de phosphates. 


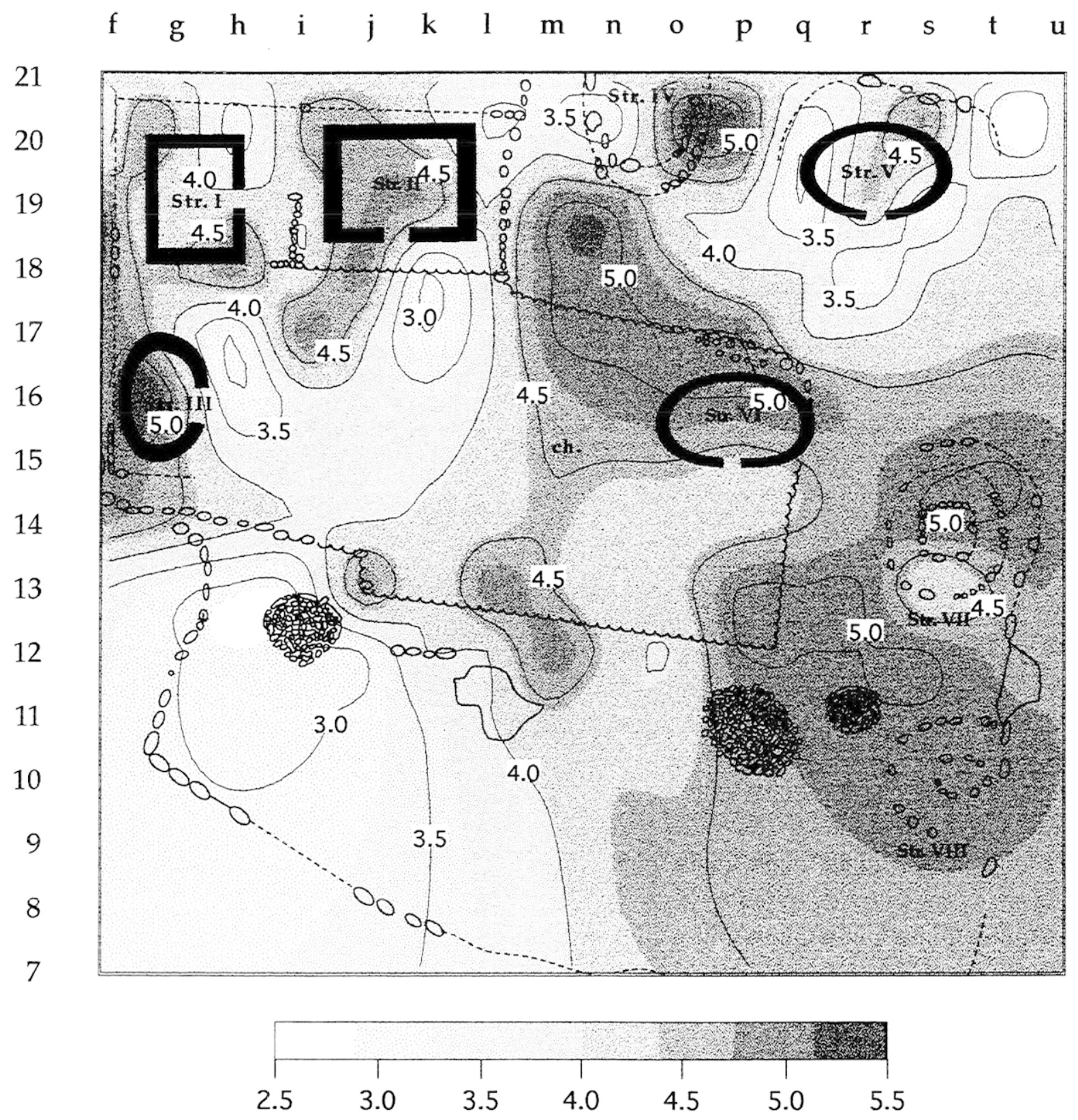

Fig. 9: La plate-forme du Cabrío, Kabah; distribution des valeurs de carbonates.

par l'ajout d'un remblai de pierres retenu par les murs de la plate-forme ainsi formée et haute de $0,60 \mathrm{~m}$ à $1,40 \mathrm{~m}$. Sur la plate-forme un espace stuqué servait d'aire de captage des eaux de pluies qu'un léger dénivelé dirigeait vers la citerne. Cette eau, ainsi recueillie durant la saison des pluies devait suffire aux besoins des habitants durant la saison sèche. Sur cet espace, étaient situées les structures domestiques I, II, III et VI. A l'est de cet espace, l'autre section de la plate-forme n'était pas stuquée et devait être utilisée pour la réalisation de tâches contaminantes. Elle était bordée par deux petites structures circulaires probablement rituelles, les structures VII et VIII. Enfin, la section située à l'extérieur de la plate-forme et les structures IV et V, plus problématiques, seront discutées ultérieurement. L'absence de stratigraphie ne nous a pas permis de déterminer une succession d'occupation, nous pouvons seulement dire que toutes les structures étaient occupées et abandonnées durant l'horizon Cehpech (750 - 1000 ap. J. -C.) et, plus exactement, durant une période intermédiaire. Un point important ici, aucune contamination matérielle postérieure n'a été mise au jour.
Les fouilles par décapage horizontal, réalisées dans et autour des structures, visaient à atteindre les surfaces d'occupation. Des sondages ont vérifié l'absence de superposition de sols. Une fois les superficies d'occupation dégagées, les échantillons ont été prélevés tous les mètres dans les structures, tous les deux mètres à leurs abords immédiats et tous les quatre mètres dans les espaces plus éloignés. Comme dans le cas précédent, la répartition des valeurs chimiques (de phosphates, de carbonates, de pH, d'albumine, d'acides gras) et les variations de couleur ont été mises en parallèle avec celle des données archéologiques (données de fouilles et répartition du matériel). Mais ici, elle a pu être confrontée à une analyse spatiale d'un matériel céramique abondant et significatif (fig. 7). La répartition des valeurs chimiques a été représentée à deux échelles différentes. Une offre une vision générale de la plateforme (fig. 8 à 11, 13 et 14) mais certains détails n'apparaissent pas; l'autre, plus précise, permet de mettre en évidence des aires d'activité à l'intérieur des structures (fig. 12).

L'espace résidentiel est tout d'abord une aire de 
captage des eaux de pluies vers la citerne. Comme le montre les faibles valeurs de phosphates, les habitants de la plate-forme s'efforçaient de le maintenir propre (fig. 8). Mais, face aux structures II et III, se seraient déroulées des activités ayant usé le revêtement de stuc; comme le montre les faibles taux de carbonates qui sont également associés et à une baisse du pH (fig. 9 et 11). Les quatre structures situées sur cet espace présentent une superficie habitable et un matériel céramique de nature domestique. $\mathrm{Ce}$ sont les vestiges de bâtiments construits selon un même principe. Il ne reste que les assises de pierres des murs, leurs parties supérieures et les toits, faits de matériaux périssables, ont disparu; leur sol était stuqué. Cependant, des différences de dimensions, de factures ainsi que la céramique associée et les composants chimiques imprégnés dans les sols suggèrent des variations fonctionnelles et précisent des aires d'activité.

Tout d'abord, la str. II se distingue; elle semble avoir été un logis. C'est la plus grande structure, la mieux élaborée, la seule à être située sur une terrasse et elle occupe une position stratégique. La céramique est représentée par une grande abondance de jarres striées utilisées pour la conservation. Mais, conserver n'était pas l'unique fonction réalisée dans cette structure et la polyvalence des logis est attestée dans les habitations actuelles comme dans des habitations préhispaniques fouillees dans d'autres sites mayas (Manzanilla ed., 1987; Manzanilla et Barba, 1990; Arnauld, 1989). En effet, plus en détail, la distribution du matériel céramique et celle des valeurs chimiques indiquent diverses aires d'activité (fig. 12). Dans l'angle nord-est, les valeurs de $\mathrm{pH}$ augmentent suffisamment pour suggérer la présence d'un foyer. A proximité des concentrations de phosphates (fig. 12), d'albumine et d'acides gras (fig. 13 et 14) confirmeraient la réalisation d'activités culinaires et de consommation dans la moitié nord de la structure, qu'atteste également l'abondance de plats et de bols et la présence de bassins. L'aire de repos pourrait être située dans l'angle sud-est, dépourvu de vestiges maté-

21

18

17

$\begin{array}{llllllllllllllll}\mathrm{f} & \mathrm{g} & \mathrm{h} & \mathrm{i} & \mathrm{j} & \mathrm{k} & \mathrm{l} & \mathrm{m} & \mathrm{n} & \mathrm{o} & \mathrm{p} & \mathrm{q} & \mathrm{r} & \mathrm{s} & \mathrm{t} & \mathrm{u}\end{array}$
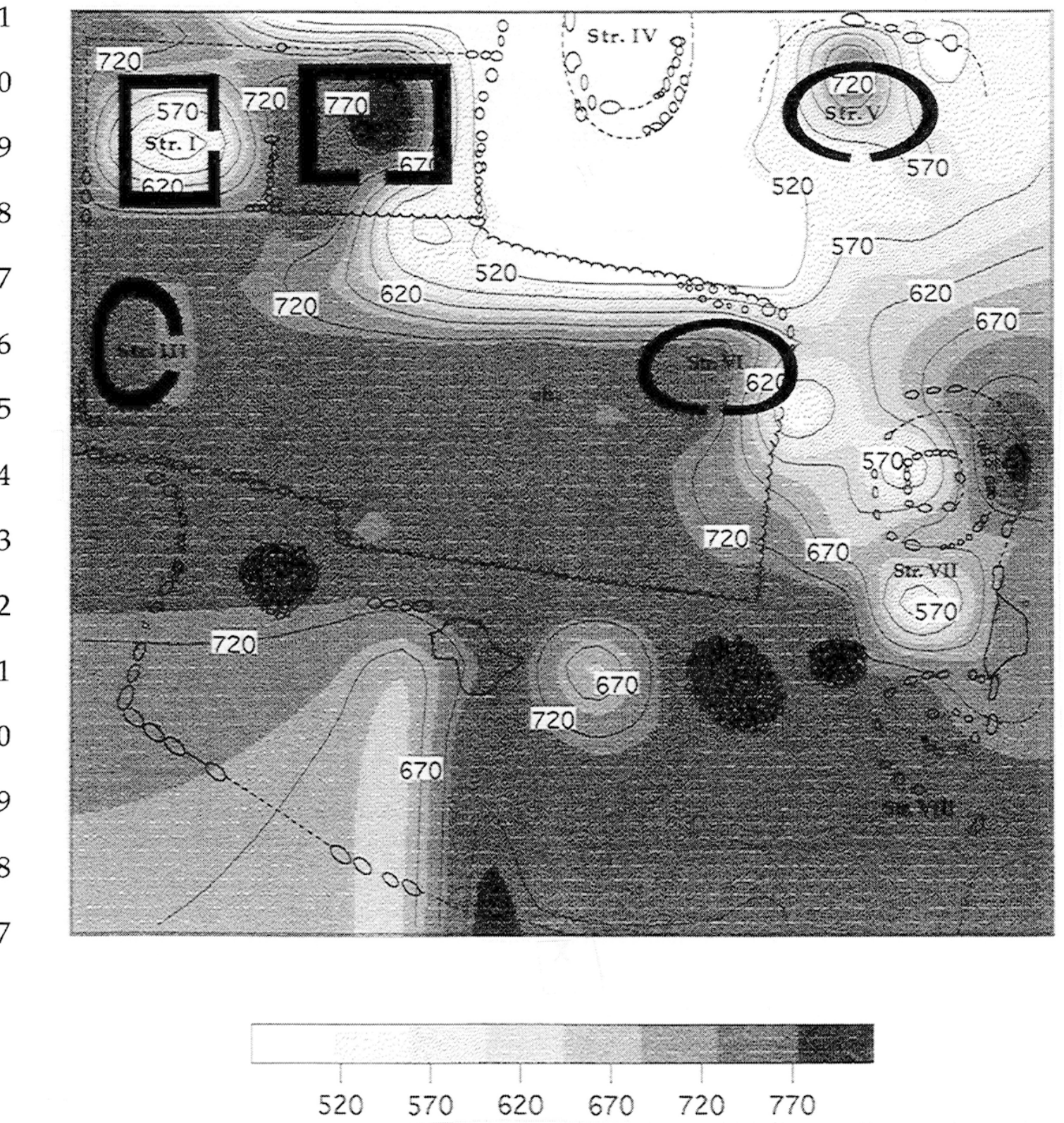

Fig. 10: La plate-forme du Cabrío, Kabah; distribution de couleur. 

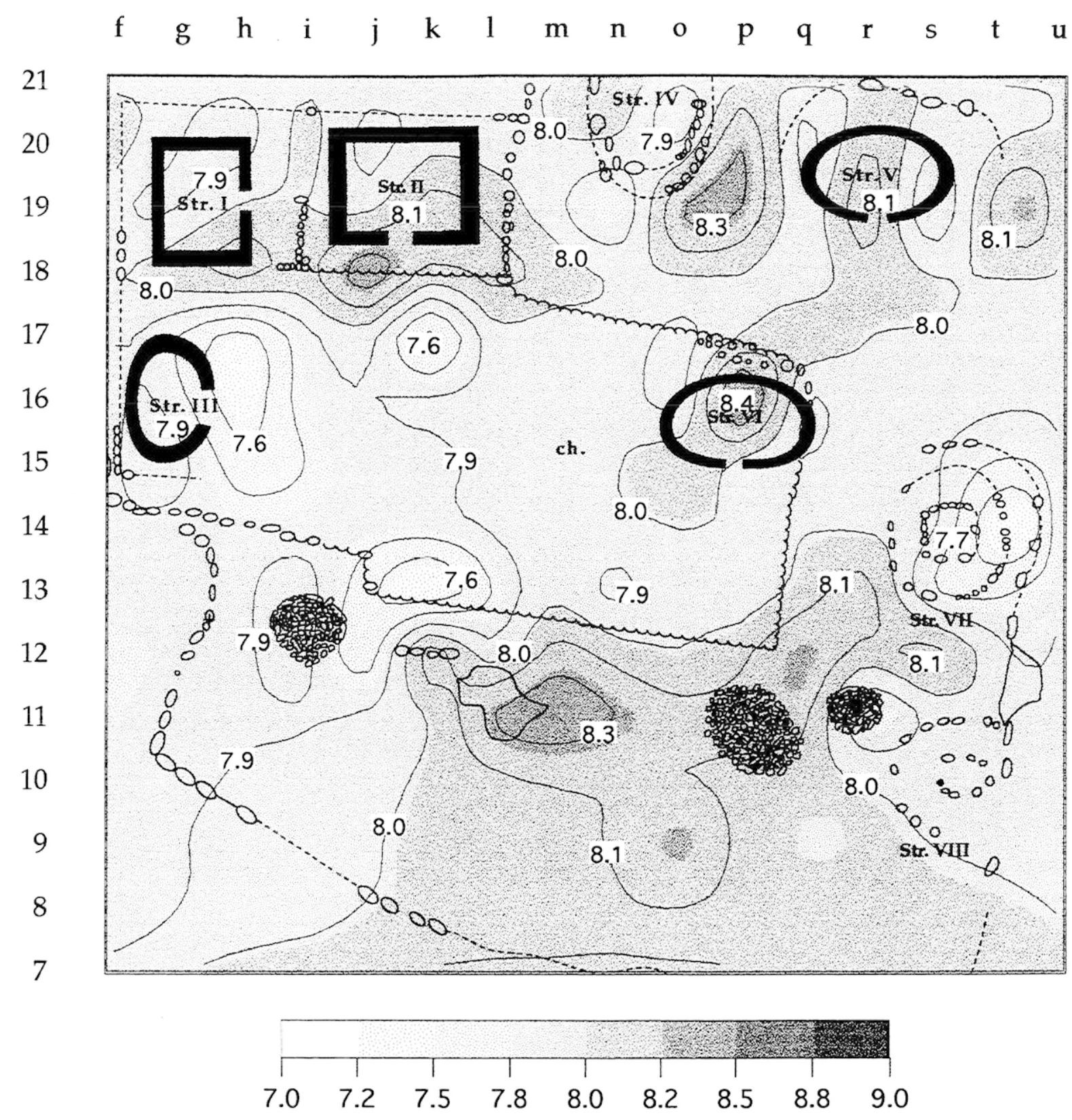

Fig. 11: La plate-forme du Cabrío, Kabah; distribution des valeurs de pH.

riels et chimiques. A cet endroit et au centre de la structure, les tessons d'au moins cinq jarres ont été mis au jour (fig. 7). Il semble que ces jarres étaient suspendues ou posees sur les poutres du toit: les tessons ne présentaient aucune configuration et l'augmentation des valeurs chimiques indiquent que cet endroit n'était pas protégé. Des aires de conservation seraient représentées par des fonds de jarres et de bassins posés sur le sol. A l'extérieur, dans l' angle nord-ouest, les vestiges de deux bassins et de deux jarres ont été mis au jour. Ils ont pu être déposés à cet endroit afin de collecter l'eau de pluie provenant des toits. La concentration de phosphates associée indique également que des activités contaminantes y étaient réalisées. La localisation de ces activités au bord de la plate-forme facilitait le maniement et le rejet d'eaux sales sans contaminer l'aire de captage.

Bien qu'aucune valeur $\mathrm{pH}$ ne soit assez élevée pour indiquer l'emplacement d'un foyer, la str. I présente toutes les caractéristiques d'une cuisine. Moins bien construite que la str. II, c'est le bâtiment qui lui est le plus proche. En termes généraux, la céramique est représentée presque exclusivement par des bols et des bassins et le sol présente des taux de pH élevés. Les pierres supérieures du remblai brûlées et la cendre écoulee dans le même remblai indiquent, dans l'angle sud-ouest, la présence d'un foyer. Dans le détail, la distribution des valeurs chimiques et des donnees archéologiques se complètent parfaitement. A l'ouest, la présence d'acides gras, à proximité du foyer, confirme l'existence d'une aire de préparation, une jarre striée indiquerait une aire de conservation et une grande quantité de bols, une aire de rangement (fig. 12). L'extrémité est de la structure se distingue par la présence d'acides gras, d'albumine et par de fortes valeurs de phosphates qui suggèrent que les repas y étaient consommés. Au centre, des bassins dans lesquels auraient été conservés des produits solides ou de l'eau, auraient protégé le sol de la contamination. L'absence de fortes valeurs de $\mathrm{pH}$ peut être relative à la réfection du sol au niveau du foyer, comme l'indique les changements de couleur; dans ce cas, cette structure aurait changé de fonction avant son abandon et au moment de la construction de la str. VI. Ou bien, des perturbations post- 

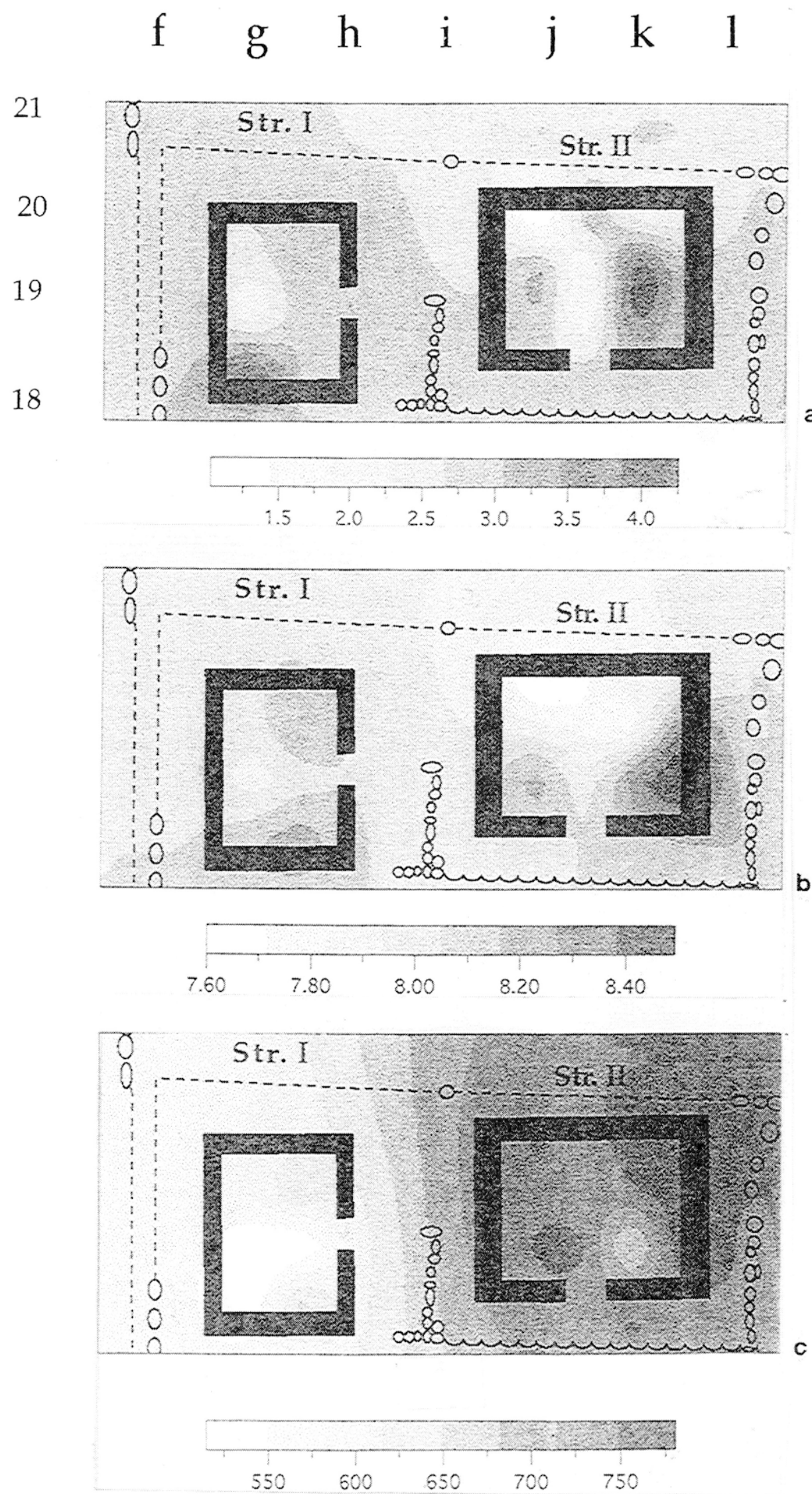


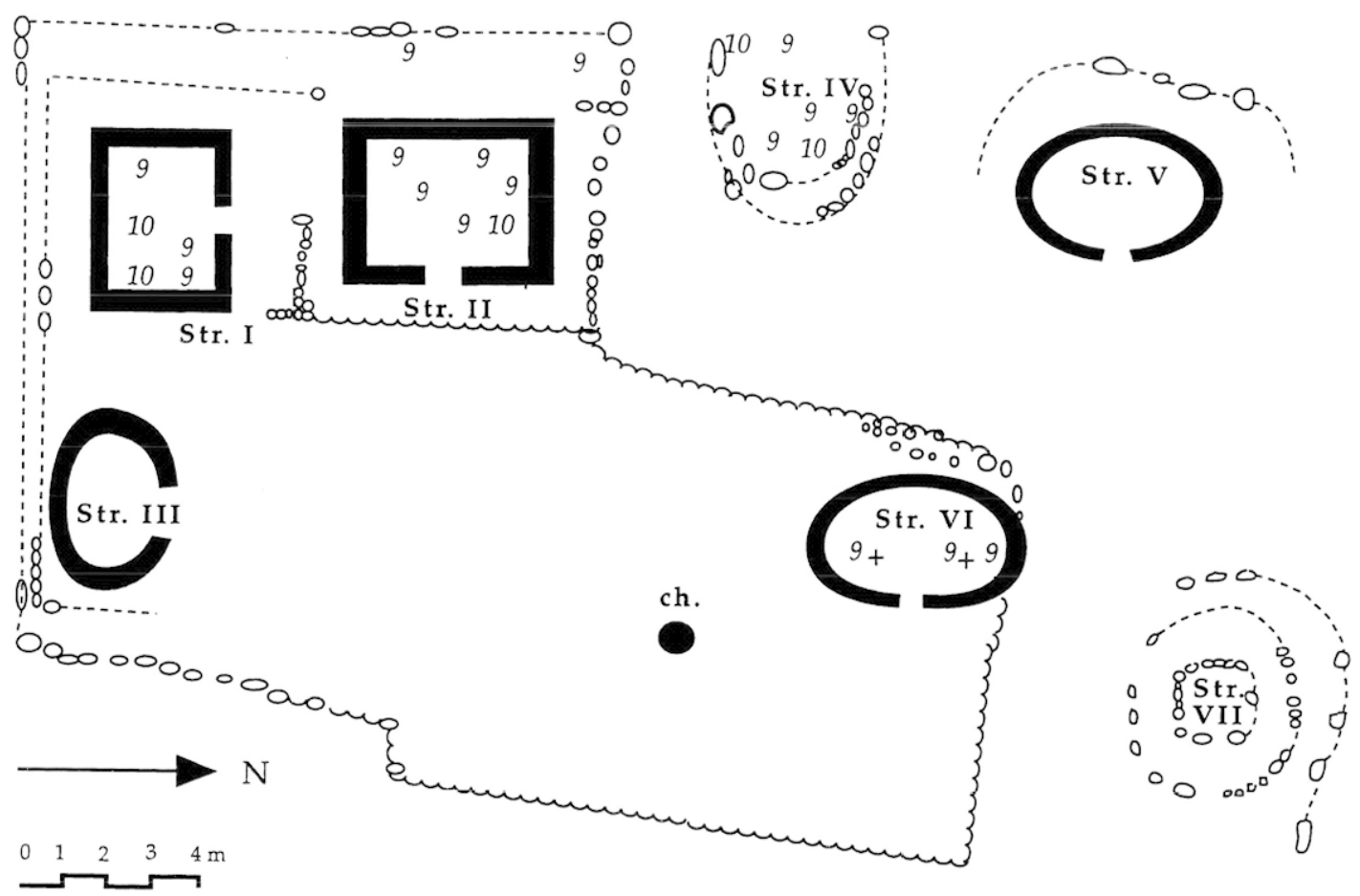

Fig. 13: La plate-forme du Cabrío, Kabah; valeurs maximales d'albumine.

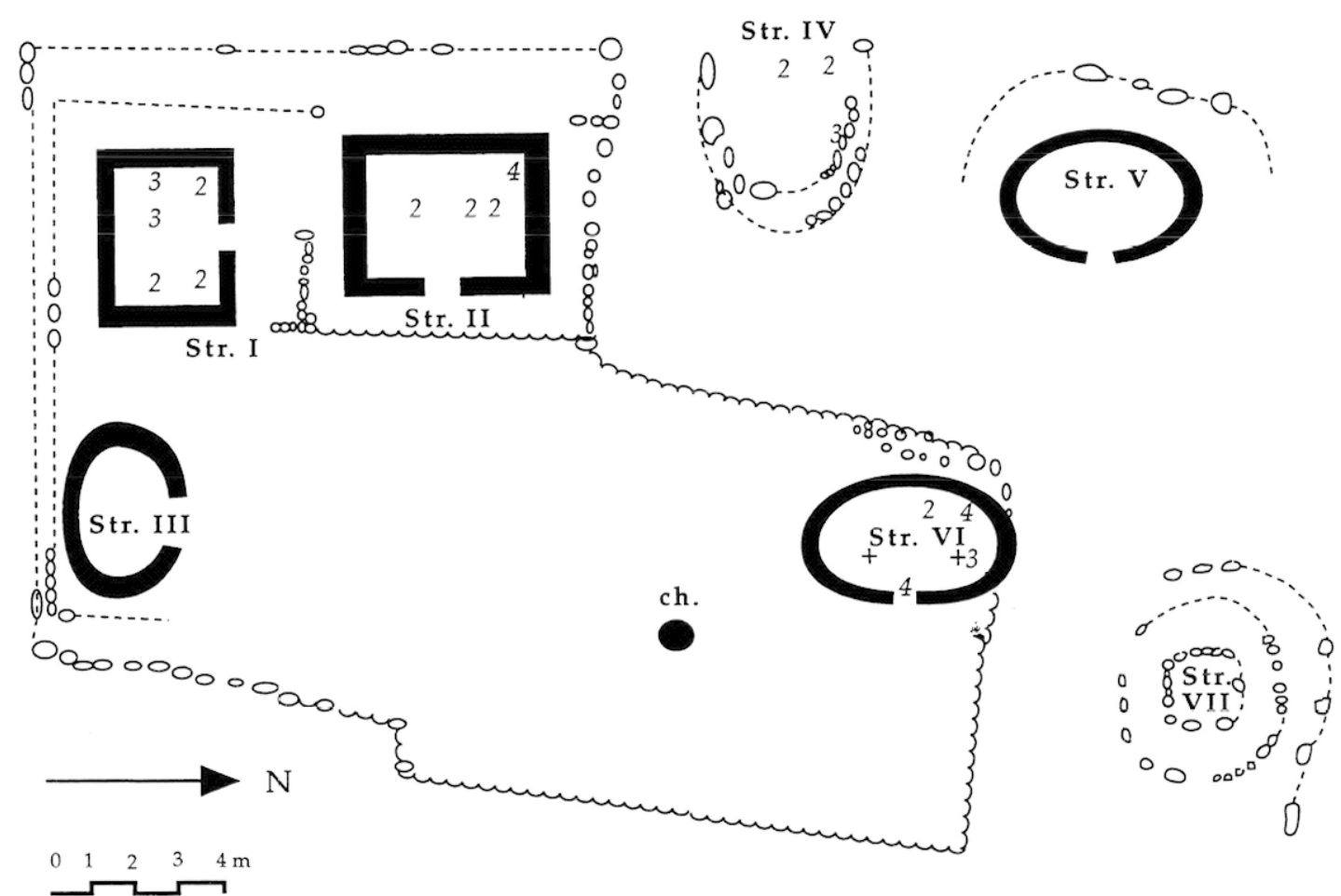

Fig. 14: La plate-forme du Cabrío, Kabah; valeurs maximales d'acides gras.

abandon n'ont pas permis de localiser la superficie d'occupation à cet endroit, au moment de la prise d'échantillons.

La répartition des valeurs chimiques observable dans la str. VI correspond à celle que nous pourrions espérer trouver dans une cuisine mais pas celle du matériel céramique trop peu abondant. Par sa localisation, indépendante des autres structures, nous pensons être en présence d'un autre logis, qui abritait également des activités de préparation et de consommation. Dans la partie ouest de cette structure, un potentiel hydrogène élevé et la présence d'acides gras indiqueraient une aire de cuisson et de préparation. La consommation des aliments serait mise en évidence par la contamination en phosphates qui apparaît dans toute la moitié ouest de la structure et par la présence d'albumine dans la partie est.

Le tiers sud de la str. III était détruit; aussi, les échantillons prélevés, trop peu nombreux, offrent peu d'informations. Notons cependant que son sol semble avoir été peu contaminé. Dans l'abside ouest, pauvre en phosphates, une jarre striée était calée sur trois pierres. 


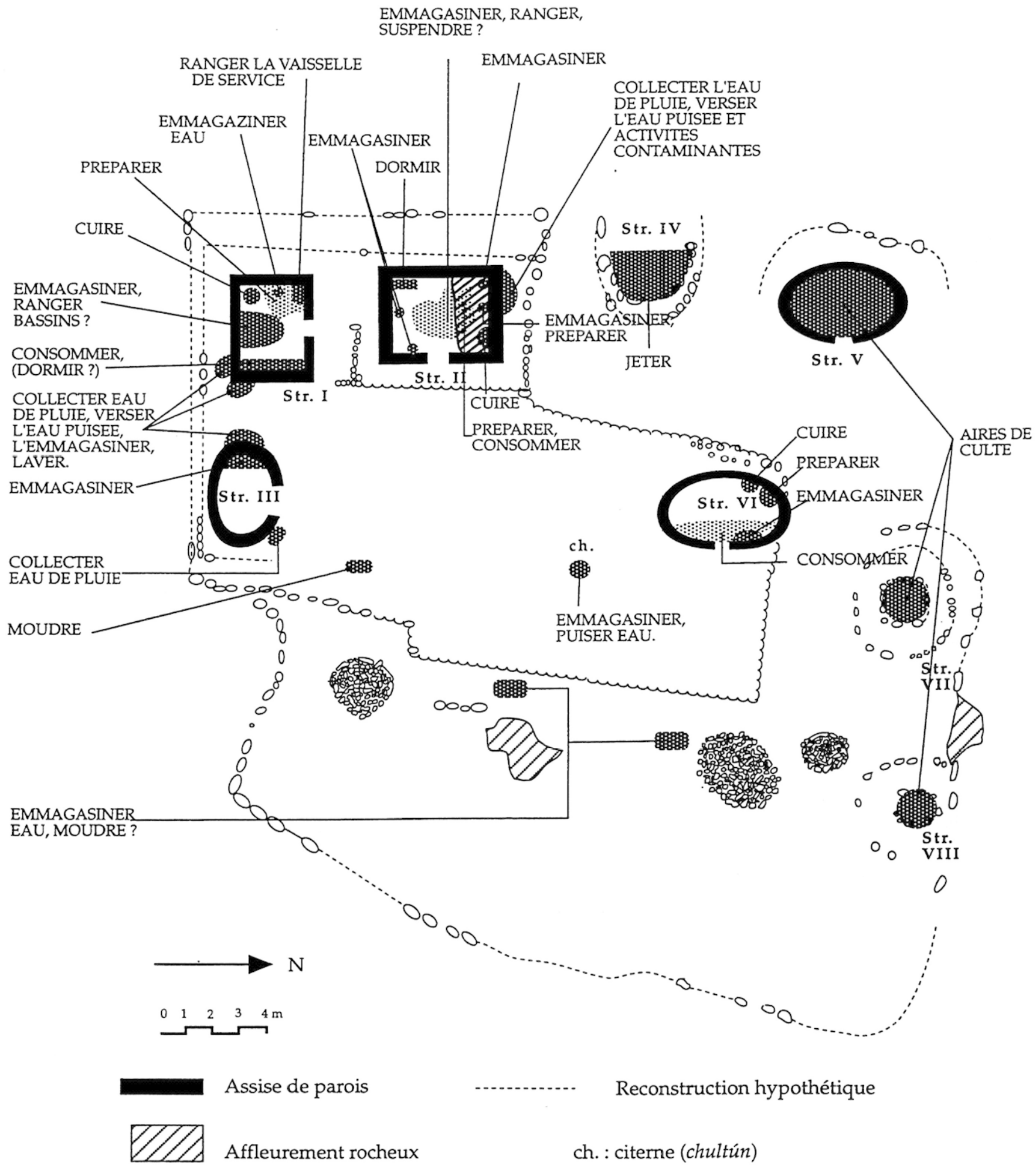

Fig. 15: La plate-forme du Cabrío, Kabah; proposition d'aires d'activités.

A l'extérieur, deux meules sont en relation avec cette structure. L'une était, au moment de l'abandon, utilisée pour moudre; l'autre, profonde, était situee sous la pente des toits afin d'y recueillir l'eau de pluie.

Les fortes valeurs de phosphates (fig. 8) apparaissant dans la section est confirmeraient que des activités contaminantes y aient été réalisées. Au nord de cette section, deux petites structures de forme circulaire semblent avoir eu une fonction rituelle. Le remblai de la str. VII contenait un ciste de pierres funéraires. La céramique abondante prélevée sur cette structure se caractérise par des formes petites et des types décorés. $\mathrm{Par}$ ailleurs, les basses valeurs de phosphates et de $\mathrm{pH}$ montrent que cette structure était régulièrement nettoyée.

Deux structures, IV et V, étaient localisées à l'extérieur de la plate-forme. Elles sont entourées d'une aire extrêmement contaminées en phosphates (fig. 8) qui suggérerait la présence de décharges. Curieusement, la str. V semble, par sa céramique et par les sépultures trouvées dans le remblai, avoir eu, au moment de l'abandon, une fonction rituelle. Comme dans la str. 
VII, les taux de phosphates enregistrés dans cette structure baissent par rapport à ceux de l'espace extérieur.

La str. IV, de forme semi-elliptique se divise en deux parties, l'une est plane et recouverte de petites pierres, l'autre, concave, est recouverte de grandes pierres plates. Dans cette partie, les fouilles ont mis en évidence des pierres brûlées et de la cendre et un très grand nombre de tessons. Au moment de l'abandon et selon les composants chimiques analysés, cette structure semble avoir été utilisée comme décharge. Les phosphates (fig. 8) présentent de fortes concentrations sur toute la structure sauf dans l'angle nord-est qui était protégé par une grande pierre. Les fortes valeurs d'albumine et d'acides gras (fig. 13 et 14) n'étant pas associées à des éléments indiquant des aires de préparation ou de consommation confirmeraient que cette structure ait servi de décharge. La présence de cendres et de pierres brûlées ainsi que des traces de chauffe sur un certain nombre de tessons indiqueraient que les rejets auraient été brûlés mais les faibles valeurs de $\mathrm{pH}$ suggèrent que cette activité a été occasionnelle ou déplacée à l'extérieur, au nord-est (fig. 11).

\section{RESULTATS ET PERSPECTIVES}

Après des études de sol d'habitations occupées (Barba et Ortiz, 1992), l'analyse de l'habitation de Muxucuxcab confirme, dans un contexte archéologique relativement bien connu, que des activités domestiques se répétant en un même lieu contaminent le sol. Elle démontre également, après une courte période d'abandon, que les composants chimiques déposés se conservent et qu'il est possible de comprendre l'articulation spatiale de l'espace et d'identifier des aires d'activité à travers leur répartition et celle de la culture matérielle. L'étude de la plate-forme du Cabrío présentait une problématique différente; la vie domestique préhispanique est peu connue mais le matériel céramique y était abondant. Dans ce cas, nous avons pu apprécier la richesse d'informations que présentent la corrélation d'une analyse chimique et d'une étude archéologique. Cette étude, comme celles réalisées dans la Casa de Oztoyahualco (Teotihuacan), dans l'Enceinte de los Caballeros Aguila (Templo Mayor, Mexico) et dans deux unités d'établissement de Cobá (Ortiz et Barba, 1993; Ortiz et al., 1994; Barba et Manzanilla, 1987; Manzanilla et Barba, 1990) démontrent que les activités humaines préhispaniques peuvent être abordées à travers les résidus qui restent imprégnés dans les sols.

La distribution des composants chimiques imprégnés dans les sols des deux habitations présentées ici confirment la division de l'espace domestique en espaces et sections d'activités dans lesquels peuvent être identifiés des aires d'activité (fig. 6 et 15). Les aires de consommation et de préparation sont les plus facilement identifiables; les aires de conservation de denrées solides ou d'eau se traduisent par l'absence de contamination. Les décharges, certaines cultures intensives et l'élevage enrichissent le sol en phosphates. Dans l'unité d'habitation de Muxucuxcab, d'autres aires d'activité apparaissent (lavoir, fours de terre, latrines) mais elles n'ont pas encore été identifiées dans des contextes préhispaniques.

D'un point de vue culturel, les travaux réalisés sur la plate-forme du Cabrío, comme ceux réalisés à Cobá amènent des informations et ouvrent des perspectives encourageantes. Dans les Basses Terres septentrionales mayas, l'habitation est, le plus souvent, connue à travers des travaux de prospection aussi parle-t-on générale- ment du logis et de ses annexes. Les travaux cités cidessus, apportent des données nouvelles sur la fonction des bâtiments, leur polyvalence, sur l'emplacement des aires d'activité qu'il s'agirait de confirmer et de compléter afin de mieux comprendre la vie quotidienne, le groupe domestique et son organisation. Aussi, pour confirmer les interprétations ci-dessus présentées et aller au-delà il serait souhaitable d'étendre ces recherches à de plus nombreuses unités domestiques et de vérifier si les associations mises ici en évidence se répètent et présentent une distribution comparable.

\section{REMERCIEMENTS}

Nous tenons à remercier Cristobal Crivolli (Instituto de Investigaciones Antropologicas, UNAM) qui a participe à l'analyse des échantillons, Ramón Carrasco (Centre Régional de Yucatán, INAH) pour nous avoir permis de travailler sur ce site et de publier les résultats ainsi que Sytviane Boucher (Centre Régional de Yucatán, INAH) pour son assistance lors de l'analyse céramique. Nous remercions également Eric Taladoire (Centre de Recherches en Archéologie Précolombienne), Philippe Bovin et Patricia Carot (Centre d'Etudes Mexicaines et Centraméricaines) pour leurs conseils.

\section{BIBLIOGRAPHIE}

ARNAULD, M.C 1989 - Résidences dans les sites mayas de Xcochkax et de Tonina, analyse comparative. Enquêtes sur l'Amérique moyenne; coordinateur, Dominique Michelet, 33-46, CEMCA, Mexico.

ARRHENIUS, O, 1931 - Die Bodenanalyse im Dienst der Archaeologie. Zeitschrift für Pflanzenerähnung Düngung und Bodenkaude, 10, 427-439.

BARBA, P.In, 1986 - La quimica en el estudio de áreas de actividad. In: Linda Manzanilla (ed.), Análisis de unidades habitacionales mesoamericanas y sus areas de actividad, 21-39; Instituto de Investigaciones Antropológicas, UNAM, México.

BARBA, P.L 1989 - Sugerencias para el mejor empleo de los datos de color Munsell. Antropológicas, 3, 98-107. Instituto de Investigaciones Antropológicas, UNAM, México.

BARBA, P.L. et J.Ln, CORDOVA, F, 1985 - Tecnología mava. el lavado de la ropa. Contactos, Revista de Educación en $C_{i}$ ias Basicas de Ingeneria, II, (28), 26-29, México.

BARBA, P.L. et MANZANILIA, In 1987 - Estudio de áreas de actividad.In: Linda Manzanilla (ed.), Cobá Quintana Roo:Analisis de dos unidades habitacionales mayas, 69-115, UNAM, México.

BARBA, P.L. et ORTIZ, A, 1992 - Análisis químicos de pisos de ocupación: un caso etnográfico en Tlaxcala, México. Latin American Antiquity, 3, (1), 63-82.

BARBA, L, RODRIGUEZ, R et CORDOBA, J.L., 1991 - Manual de técnicas microquímicas de campo para la arqueología. Cuadernos de Investigación, Instituto de Investigaciones Antropológicas, UNAM, México.

COOK, S.F. et HEIZER, R.F, 1965 - Studies on the chemical analisis of archaeological sites. University of California, Publication in anthropology, 2.

CRADDOCK, P, 1982 - Quand les archélogues analysent les phosphates. La Recherche, 133, 666-667.

DUNNING, N.P, 1991 - Ancient Anthrosol of the maya Lowlands a reexamination of the regional variability in the prehispanic agricultural landscape. Communication, conference on the maya agricultural and biological resource management, University of California, Riverside.

EIDT, R, 1973 - A rapid chemical test of archaeological site surveying. American Antiquity, 38, 206-210.

FAO-UNESCO, 1975 - Soils map of the world, 1: 5000000, III, Mexico and Central America, UNESCO, Paris.

GUILLERAULT, P. et BAZILE, F, 1987 - Le dosage des phosphates en archéologie: la méthode potentiométrique. Bull. Soc. préhist. française, 84, (3), 73-74.

HAMOND, F.W, 1983 - Phosphate analysis of archaeology sediments. In: Landscape archaeology in Ireland, B.A.R., B.S., 116, $47-80$. 
KEELEY, H.C.M., 1981 - Recent work using phosphorus analysis in archaeological prospection. Rev. Archéometrie, 5, 89-95.

MANZANILLA, Lo, 1987 - Cobá Quintana Roo: Analisis de dos unidades habitacionales mayas. UNAM, Mexico

MANZANILLA, $L$ et BARBA, $L_{m} 1990$ - The study of activities in classic households: two case studies from Coba and Teotihuacan. Ancient Mesoamerica, 1, 41-49.

MEJIA, E. et BARBA, L, 1998 - El análisis de fosfatos en la arqueología: historia y perspectivas; Anales de Antropologia, 25, 127-147, Instituto de Investigaciones Antropológicas, UNAM, México.

ORTIZ, A. et BARBA, Lo, 1993 - La química en el estudio de áreas de actividad. In: Linda Manzanilla (ed.) Anatomía de un conjunto residencial teotihucano en Oztoyohualco, I, chap. XII. UNAM, Mexico.
ORTIZ, A.L BARBA, P.I, LOPEZ LUJAN et LINK, KF, 1994 Stuccoed Floors a resource for the study of ritual activities. The case of Templo Mayor, México. Communication, Issues in Art and Archaeology, N, Cancún, Mexique.

PIERREBOURG, F. de, 1989 - El espacio doméstico maya: una mirada arqueologica sobre el présente: Proposición de un método. TRACE , 16, 31-42, CEMCA, México.

PIERREBOURG, F. de, 1994/95 - L'espace domestique maya: une étude ethnoarchéologique au Yucatan. Ms. These de Doctorat, Paris I.

PROVAN, D.M.J, 1971 - Soil phophate analysis as a tool in archaeology. Norvegian archaeological Review, 4, 37-50.

SMYTH, M.P., 1990 - Maize storage among the puuc maya. Ancient Mesoamerica, 1, 51-69. 